\title{
Industria porcina china, sistema agroalimentario global y crisis ambiental. Reflexiones a partir del caso argentino
}

SOL MORA*

Artículo recibido: 30 de junio de 2021

Artículo aprobado: 22 de noviembre de 2021

Doi: https://doi.org/10.12804/revistas.urosario.edu.co/desafios/a.10764

Para citar este artículo: Mora, S. (2022). Industria porcina china, sistema agroalimentario global y crisis ambiental. Reflexiones a partir del caso argentino. Desafíos, 34(1), 1-32. https:/ / doi.org/10.12804/revistas.urosario.edu.co/desafios/a.10764

\section{Resumen}

El severo impacto ambiental de los actuales patrones de producción y consumo de alimentos y la pandemia de COVID-19 otorgaron una inusitada relevancia a la transformación del sistema agroalimentario a nivel local y global. Este trabajo explora las implicancias de las acciones de China para la protección de su industria porcina tras la epidemia de peste porcina africana, en la trayectoria del sistema agroalimentario global. Para ello se estudió el proyecto de instalación de granjas porcinas en Argentina negociado en 2020. Mediante un diálogo del enfoque neogramsciano de la economía politica internacional con los estudios agrarios críticos, se argumenta que esa iniciativa demuestra que, pese a los efectos ambientales y sanitarios, el interés de China no es transformar su modelo de producción porcina, sino trasladarlo hacia nuevos territorios. De ese modo, China reconstruye su liderazgo porcino y afirma su poder dentro del modelo agroalimentario hegemónico.

* Instituto de Investigaciones Políticas (IIP)- CONICET- Escuela de Política y Gobierno, UNSAM. Correo electrónico: smora@unsam.edu.ar ORCID: https://orcid. org/0000-0002-8237-6938 
Palabras clave: industria porcina; China; sistema agroalimentario; Argentina; peste porcina africana; ambiente.

\title{
The Chinese Pork Industry, the Global Agri- Food System and the Environmental Crisis. Reflections Based on the Argentine Case
}

\begin{abstract}
The severe environmental impacts of the current food production and consumption patterns and the covid-19 pandemic have given an unprecedented relevance to the transformation of the agrifood system at the local and global levels. This article explores the implications of China's actions seeking to protect its pork industry following the African Swine Fever epidemic on the trajectory of the global agrifood system. To this end, it studies the project to installpigfarms in Argentina negotiated in 2020. Drawing on a dialogue betwenn the neo-Gramscian approach to International Political Economy with critical agrarian studies, I argue that this initiative shows that, despite environmental and health consequences, China's interest is not in transforming its pig production model, but in transferring it to new territories. As a result, China rebuilds its pork leadership and thus reaffirms its power within the begemonic agrifood model.
\end{abstract}

Keywords: Pork industry; China; agrifood system; Argentina; African Swine Fever; environment.

\section{Indústria suína chinesa, sistema agroalimentar global e crise ambiental. Reflexões do caso argentino}

\begin{abstract}
Resumo
O severo impacto ambiental dos atuais padrões de produção e consumo de alimentos e a pandemia COVID-19 deram uma relevância incomum à transformação do sistema agroalimentar em nivel local e global. Este artigo explora as implicações das ações da China para a proteção de sua indústria suína após a epidemia de peste
\end{abstract}


suína africana na trajetória do sistema agroalimentar global. Para isso, estuda-se o projeto de instalação de granjas de suínos na Argentina negociado em 2020. Por meio de um diálogo de abordagem neogramsciana da economia política internacional com os estudos agrários críticos, argumenta-se que esta iniciativa mostra que, apesar do aspecto ambiental e implicações sanitárias, o interesse da China não consiste em transformar seu modelo de produção de suinos, mas em transferi-lo para novos territórios. Desta forma, a China reconstrói sua liderança no mercado suino e afirma seu poder dentro do modelo agroalimentar hegemônico.

Palavras-chave: suinocultura; China; sistema agroalimentar; Argentina; peste suina africana; meio ambiente.

\section{Introducción}

Existe amplia evidencia de que los actuales patrones alimentarios y el modo de producción de alimentos ejercen una contribución significativa al cambio climático. De acuerdo a la Organización de Naciones Unidas para la Agricultura y la Alimentación (FAO), en el periodo 20072016, el sistema agroalimentario fue responsable de entre el 21 y el $37 \%$ de las emisiones de gases de efecto invernadero (GEI), asociadas a actividades agrícolas y ganaderas, cambios en el uso de la tierra y el procesamiento, comercialización, consumo, desperdicio y desecho de alimentos (FAO et al., 2020). Una actividad particularmente destacada por su impacto climático es la ganadería, ya que representa el 14,5\% del total de emisiones de GEI a nivel global (FAO, 2014). Con todo, la producción agroalimentaria posee otras implicancias ambientales negativas. Entre ellas se encuentran la degradación de la tierra, la disminución de la calidad y disponibilidad de agua, la deforestación y la pérdida de biodiversidad.

Lo anterior pone de relieve que las prácticas agroalimentarias vigentes no solo deterioran los recursos naturales, sino que además comprometen las condiciones ambientales de las que dependen esas prácticas, lo que socava las posibilidades de producción alimentaria futura (Powers, 2021). El Panel Intergubernamental de Expertos sobre el Cambio Climático (IPCC, por sus siglas en inglés) reconoció en 2019 que la seguridad alimentaria se verá altamente afectada por el cambio climático. Las variaciones en la temperatura y los patrones 
de precipitación y la frecuencia de eventos climáticos extremos afectarán la productividad agrícola y alterarán la calidad nutricional de los alimentos, lo que se verá intensificado por la difusión de pestes y enfermedades. También se prevén incrementos en los precios de los alimentos e inestabilidad en el suministro.

Las tendencias señaladas llevaron al reconocimiento que la meta de compatibilizar la producción y consumo de alimentos con la sostenibilidad exige producir transformaciones en el sistema agroalimentario (FAO et al., 2020). Con todo, la necesidad de reconstruir el sistema alimentario a nivel local y global adquirió una inusitada relevancia con la pandemia de COVID-19 por dos razones. Por un lado, se tornaron visibles los vínculos entre el sistema agroalimentario industrial global y la propagación de enfermedades: la producción ganadera intensiva, la deforestación y destrucción de hábitats y la urbanización acelerada incrementan las interacciones entre humanos y animales salvajes, $\mathrm{y}$, por ende, el riesgo de zoonosis — situaciones que fueron señaladas como causas de la enfermedad- (Giudice et al., 2020; James et al., 2021; Silva \& Barbosa, 2020). Por otro lado, la crisis alimentaria ${ }^{1}$ provocada por la pandemia se presentó como una oportunidad única para explorar otras prácticas y principios sobre los cuáles reorganizar los sistemas agroalimentarios. Reflejo de ello es la creciente discusión en torno a alternativas que contemplan la agroecología (Clapp \& Moseley, 2020), la justicia alimentaria (Sanderson Bellamy et al., 2021), la democracia alimentaria (Petetin, 2020) y la economía circular (Giudice et al., 2020), entre otras.

Con el objetivo de contribuir a esas discusiones, este trabajo explora las implicancias de las acciones de China para la protección de su industria porcina tras la epidemia de peste porcina africana (PPA), cuyo origen se rastrea al 2018, en la trayectoria del sistema agroalimentario global. Los intensos flujos de comercio e inversiones para

\footnotetext{
1 Esa crisis es el resultado de las interrupciones en la distribución de alimentos debido a las medidas de confinamiento y los contagios entre los trabajadores del sector, el incremento del hambre provocada por la pérdida masiva de empleos y las dispares dinámicas de precios a nivel local y global (Clapp \& Moseley, 2020).
} 
garantizar su seguridad alimentaria convirtieron a China en un actor clave en las dinámicas agroalimentarias globales (McMichael, 2020). Además, China ha experimentado de manera particular las tensiones entre ambiente y actividad agroalimentaria debido a las consecuencias ambientales y sanitarias derivadas del esfuerzo por alimentar a su población y abastecer su creciente consumo de carnes - especialmente de cerdo-, al tiempo que alcanza la primacía global en la producción porcina con una limitada disponibilidad doméstica de tierras cultivables y agua (Trápaga Delfín, 2017; Zhang \& Cheng, 2016). En este marco, las negociaciones de un proyecto de inversión para la instalación de granjas para la producción porcina a gran escala en Argentina durante 2020, cuando la lucha contra la PPA en China continuaba, son relevantes para reflexionar sobre las dinámicas en el sistema agroalimentario global en un contexto de crisis sanitaria y ambiental.

Mediante una aproximación teórica resultante del diálogo del enfoque neogramsciano de la economía política internacional con los estudios agrarios críticos, se argumenta que la epidemia de PPA expresa las debilidades inherentes al actual modelo de producción porcina industrial. Sin embargo, la amplia controversia generada en torno al proyecto porcino en Argentina demuestra que el interés de China no consiste en transformar ese modelo, sino en su traslado hacia nuevos territorios. De ese modo, se exteriorizan las consecuencias ambientales y sanitarias, mientras China reconstruye su liderazgo porcino mundial y, por lo tanto, reafirma su poder dentro del modelo agroalimentario hegemónico.

Acorde con lo anterior, se empleó una metodología cualitativa consistente en un estudio de caso. La información fue recolectada mediante el análisis de documentos, entre los que se encuentran comunicados de la Cancillería y del Ministerio de Agricultura de Argentina (MAGyP); declaraciones de funcionarios a la prensa, e informes y documentos elaborados por organizaciones ambientales y sociales, académicos y productores porcinos. Ello es complementado con informes de organizaciones internacionales vinculadas al ambiente, la salud y la producción y consumo alimentario, así como publicaciones académicas en esas temáticas. 


\section{6 / SOL MORA}

El primer apartado caracteriza el modelo agroalimentario hegemónico. Posteriormente se explica la posición China en ese modelo y se describen los rasgos centrales de su industria porcina y sus efectos ambientales. En tercer lugar, se presenta el análisis de los desafíos que plantea la PPA para la seguridad alimentaria de China. El último apartado se concentra en el proyecto porcino en Argentina.

\section{El modelo global de producción agroalimentario hegemónico}

Para comprender el proyecto porcino en Argentina es indispensable remitirse al sistema agroalimentario en que tiene lugar la iniciativa, específicamente, su rasgo definitorio: la hegemonía. De acuerdo con Robert Cox (1993), esta categoría expresa un orden fundado en valores y entendimientos que son aceptados incuestionablemente como el estado natural de las cosas por la mayoría de los actores. Aunque la coerción puede apuntalar esos significados intersubjetivos, Cox (1992) consideró los aspectos ideológicos y culturales como el factor decisivo para el establecimiento de la hegemonía. La razón es que estos fundamentan un orden en apariencia universal, que incorpora algunas demandas de los grupos subordinados, sin afectar los intereses particulares de la clase hegemónica (Cox, 1993). Por eso, la hegemonía puede ser entendida como "una conjunción coherente o acoplada entre una configuración de poder material — la imagen colectiva imperante del orden mundial (incluyendo ciertas normas) - y un conjunto de instituciones que administran el orden con una cierta apariencia de universalidad" (Cox, 2013, p. 146).

La particularidad de Cox (1993) es que abordó la hegemonía global no solo como un orden interestatal, sino además como un orden dentro de una economía mundial con un modo de producción dominante que permea todos los países, así como un complejo sistema de relaciones sociales internacionales. Por eso, definió la hegemonía mundial simultáneamente como una estructura social, económica y política que se expresa en normas universales, instituciones y mecanismos de comportamiento que recaen sobre Estados y fuerzas sociales, 
que al mismo tiempo, sostiene el modo de producción dominante (Cox, 1993).

Dentro del sistema agroalimentario, una esfera destacada para la proyección de la hegemonía fue el modelo de producción agroalimentario global que se configuró con el neoliberalismo. Mientras que el fundamento material de ese modelo es la primacía de los intereses empresariales en las decisiones alimentarias (McMichael, 2009), su soporte ideológico es la creencia en la eficiencia de los mercados para regular las relaciones socioeconómicas. Ello consolidó el abandono de la visión de la agricultura como fuente de nutrición y sustento para ser concebida como agronegocio, sujeta a los imperativos de acumulación de capital y explotación y exclusión socioambiental (Ioris, 2018). Esa idea se nutrió del discurso que posiciona a la biotecnología como la herramienta clave para eliminar el hambre mundial (Otero, 2013). Similarmente, en la producción ganadera el imperativo de eficiencia se ligó estrechamente a la mecanización y las economías de escala (Weis, 2013).

Los argumentos expuestos justificaron la centralidad que adquirieron ciertos desarrollos técnicos y organizativos en el modelo de producción agroalimentario. En materia agrícola, estos comprenden el uso intensivo de insumos industriales y biotecnologías para la producción de monocultivos a gran escala, el acaparamiento y concentración de la tierra y la expansión del papel de las empresas y el capital financiero (Gras \& Hernández, 2013). También se afianzó un modelo de ganadería industrial basado en la intensificación y la concentración de la actividad; la expulsión de los animales de las tierras de cultivo; el establecimiento de granjas industriales con métodos productivos mecanizados, y la cría de ganado en confinamiento bajo requerimientos alimentarios y farmacológicos específicos (Emel \& Neo, 2011).

Más aún, la difusión de esas ideas en un contexto de libre movilidad de capitales favoreció el dominio de un oligopolio de empresas trasnacionales sobre los diferentes eslabones de la cadena alimentaria. ${ }^{2} \mathrm{El}$

2 Ejemplo de ello es el dominio del $70 \%$ del mercado mundial de agroquímicos por Chemchina, Bayer, BASF y Corteva Agriscience y la concentración de la comercialización en ADM, 


\section{8 / SOl MORA}

complejo ganadero industrial global es representativo de ello, ya que sucesivas oleadas de fusiones y adquisiciones provocaron la concentración de la producción de insumos, la comercialización de forrajes y el procesamiento de la carne. El último sector está encabezado por JBS, Tyson Foods, Cargill, wHGroup y NH Foods, donde las primeras tres superan ampliamente a las demás en ventas (Sharma \& Schlesinger, 2017). Ese liderazgo es fortalecido por la influencia y legitimidad que adquirieron las empresas en los mecanismos de gobernanza, de modo que contribuyen a la transformación de las relaciones Estadomercado-naturaleza (Saguier \& Ghiotto, 2018).

Este modelo de producción agroalimentaria, favorecido por el proyecto de liberalización agrícola promovido por la Organización Mundial de Comercio —OMC - (McMichael, 2009), se expandió rápidamente de los países desarrollados a los países en desarrollo. La amplia adhesión que encontraron las ideas que promueven la reconfiguración de las estructuras agrícolas de los países productores de alimentos de acuerdo a los intereses empresariales encubiertos detrás de las nociones de eficiencia, crecimiento económico y disminución del hambre expresan la hegemonía de este modelo (Mora, 2021). Esa hegemonía es reforzada por una narrativa desarrollista que asimila el consumo de carne con el progreso y, por tanto, interpreta el incremento del consumo de proteínas como una transición nutricional asociada al incremento de los ingresos (Schneider, 2014). Esos conceptos legitimaron la carnificació $n$ de las dietas (Weis, 2013) y con ello el sistema agroalimentario que abastece esa demanda.

Como contrapartida, el modelo agroalimentario global no solo ignora la existencia de formas alternativas de producción. Además, minimizan los impactos en el ambiente, la salud y la seguridad y soberanía alimentaria de actividades que involucran enormes cantidades de tierras, agua, fertilizantes, agroquímicos, combustibles fósiles, desechos tóxicos, pérdidas de nutrientes y emisiones de GEI (Weis, 2013).

Bunge, Cargill, Louis Dreyfus y China National Cereals, Oils and Food Stuffs Corporation [COFCO] (ETC GROUP, 2019). 


\section{La industria porcina china}

El dinamismo de la industria porcina asentó el liderazgo global de China en ese sector. Con una producción que en 2016 equivalió a casi la mitad del total mundial (FAO, 2017); en China el 80\% de la producción de cárnicos corresponde a la carne de cerdo (Trápaga Delfín, 2017). También encabeza el consumo total y per cápita de cerdo y derivados, con niveles que en 2014 superaron el 50\% del total global. Además, hasta el 2018, China poseía la mitad de la población porcina del globo (FAO, 2017). En simultáneo, es el primer productor global de forrajes (Fok \& Hui, 2017).

Esa primacía en la producción y consumo de carne de cerdo es inescindible del papel que China desempeña en el modelo agroalimentario hegemónico. Al respecto, este trabajo asume que antes que desafiar el dominio del capital transnacional sobre las cadenas alimentarias, China aspira a crear espacio para una mayor participación de su agronegocio en el sistema agroalimentario global. De esa forma, sus inversiones agroalimentarias en el exterior, entre ellas en el sector porcino, representan y facilitan la expansión del modelo productivo hegemónico.

El interés de China por mejorar su posición en el modelo agroalimentario global se enraíza en su enfoque hacia el desarrollo agrícola desde 1990, orientado a la construcción de un robusto sector de agronegocios doméstico que, bajo el liderazgo de las empresas nacionales, contribuya al desarrollo nacional y al acceso a recursos y mercados en el exterior (Schneider, 2017). Ese objetivo, que estuvo guiado por los supuestos de modernización agrícola compartidos con los países occidentales (Karavas \& Brincat, 2016), allanó el camino hacia el liderazgo global chino en la producción y consumo porcino. Aunque esa trayectoria se inició con la descolectivización y la liberalización selectiva del sector agrícola desde 1978, esta fue reforzada en las dos décadas siguientes mediante el estímulo al desarrollo de la industria ganadera doméstica, la industrialización de la producción y el fomento al consumo entre los sectores urbanos (Lander et al., 
2020). El resultado de esas políticas fue una explosión en la producción y el consumo de cerdo, así como una transformación radical de la estructura del sector.

Por un lado, el alza del consumo tras las reformas modificó radicalmente la tradición alimentaria china, en la que la producción de carne porcina era limitada y su consumo era racionado y tratado como un alimento muy preciado (Zhou et al., 2012). Cabe añadir que los cerdos han tenido una importancia milenaria en la cultura china, era usual que cada familia poseyera un cerdo para convertir sus residuos en fertilizantes (Trápaga Delfín, 2017).

Por otro lado, el crecimiento de la producción implicó la ampliación de la escala de las granjas. Las granjas familiares de traspatio, con un promedio de 5 cabezas, perdieron su exclusividad en el abastecimiento del mercado chino desde 1980 con el surgimiento de granjas especializadas, con decenas o incluso cientos de cabezas. En paralelo, las políticas fiscales para responder al alza en la demanda estimularon el desarrollo de granjas a gran escala para la cría de miles a millones de cerdos (Qiao et al, 2016). Como resultado, de 2007 a 2015, la producción de las granjas de traspatio se redujo de un 74 a un $57 \%$ del total. En ese periodo la producción en granjas a gran escala y especializadas ascendió a un $10 \%$ y un $30 \%$ del total, respectivamente (Fok \& Hui, 2017).

La concentración de la producción estuvo acompañada por una transformación técnica y organizativa hacia la producción industrial. Esta incluyó la importación de razas de cerdo más magras y la adopción de forrajes que acortaran el ciclo de producción. Asimismo, la operación concentrada de alimentación animal se convirtió en el corazón de la industria porcina (Lander et al., 2020). Este sistema confina miles de animales para acelerar la producción de carne con un uso limitado del suelo, lo que resulta esencial para la seguridad alimentaria de China, puesto que solo dispone del $9 \%$ de la tierra cultivable del mundo (Zhang \& Cheng, 2016). Por esa razón, las importaciones de soja y maíz, fundamentales para la elaboración de piensos, son otro pilar de 
la industria porcina. De ahí que la primacía de China en la producción de cerdo se sustente en su posición como primer consumidor e importador mundial de soja, lo que a su vez transformó los mercados y los flujos de alimentos de y en todo el mundo.

Otra pieza central para afianzar la industria porcina fue el apoyo gubernamental a empresas consideradas estratégicas para la modernización agrícola (Schneider, 2017). El ejemplo paradigmático en el sector porcino es WH Group, que realizó la mayor adquisición china de una empresa alimentaria estadounidense tras comprar Smithfeld Food. Esa operación convirtió a WH Group en la mayor empresa porcina del mundo (Gooch \& Gale, 2018).

No obstante, el vertiginoso desarrollo de la industria porcina de China fue acompañado por una amplificación de los problemas ambientales. Estos se originaron, primero, en el estiércol de las granjas industriales. Además de incrementar las emisiones de metano y óxido de nitrógeno, dos GEI, esos desechos contaminan severamente el agua. La difusión de los fertilizantes químicos, la proximidad de las granjas a las ciudades y su desconexión con la producción de forrajes limitó el tradicional empleo del estiércol como fertilizante, lo que tornó habitual que este fuera arrojado sin tratamiento en lagos y ríos (Lander et al., 2020; Zhang et al, 2021). Es de anotar que el censo nacional sobre la fuentes de contaminación en China de 2010 y su actualización en 2020 identificaron a la producción ganadera como la principal fuente de descarga de nitrógeno y fósforo en los cursos de agua (Bai et al., 2019; Xinzhen, 2020). Esto originó frecuentes brotes de algas verdes azuladas o cianobacterias que comprometen la calidad del agua (Jin, 2010).

Por otra parte, en suelos fertilizados con estiércol también se detectó contaminación con metales pesados, como zinc y cobre, que son incorporados como aditivos en los piensos (Bai et al., 2019), lo que llevó al Ministerio de Agricultura de China a fijar un límite en el uso de esos metales (Zhang et al, 2021). Otra fuente de preocupaciones asociada a la actividad porcina es el excesivo empleo de antibióticos para enfrentar el hacinamiento de animales genéticamente similares en deficientes 


\section{I SOL MORA}

condiciones higiénicas y sanitarias (Verzeñassi et al., 2020). En suelos y aguas próximos a las granjas porcinas fueron hallados genes resistentes a los antibióticos (Zhu et al, 2013; Bai et al 2019). Las bajas defensas de los cerdos hace que los trabajadores deban sanitizarse e incluso hacer cuarentena antes de entrar en contacto con ellos (Lander et al., 2020).

En adición, la degradación del agua por los desechos de animales enfermos o con presencia de antibióticos genera graves riesgos para la salud humana. Como fue señalado, esos peligros se incrementan por las falencias en el manejo de los residuos de la actividad, como lo ejemplifican los 16000 cerdos muertos a causa de una epidemia en 2013, que fueron arrojados al río Huangpu, que abastece de agua a Shanghái (Lü, 2013; Trápaga Delfin, 2017). La salud humana puede verse comprometida asimismo por el ingreso de los residuos de antibióticos o microorganismos que generaron resistencias a la cadena alimentaria (Schoenmakers, 2020; Zhang et al, 2021).

La presencia de problemáticas similares en la producción porcina de otros países (Yeboah et al, 2009; Pedreño Cánovas et al., 2021) reivindica la relevancia del enfoque Una Salud, que enfatiza la convergencia y estrecha interconexión entre la salud humana, animal y ambiental (FAO et al., 2008). Esta noción reconoce el riesgo que supone la difusión de enfermedades que emergen de la interacción entre humanos y animales. Por esa razón, rechaza las respuestas sectoriales y batalla por una aproximación holística a la prevención y control de enfermedades epidémicas y zoonóticas, con la participación de múltiples actores a diferentes escalas en beneficio de los humanos, los animales y la biodiversidad (OIE, s.f.).

Si bien el gobierno chino incorporó regulaciones ambientales a su actividad porcina, estas no alteraron los aspectos centrales del modelo productivo. Prueba de ello es que en 2015 se prohibió la producción ganadera en regiones del sur del país, con abundantes cursos de agua, mientras se promovió la transferencia de la actividad hacia provincias del sudoeste y el noreste. Con todo, debido al déficit de inversiones para el manejo de los excrementos, las granjas relocalizadas construyeron lagunas que contaminan el aire, lo que demuestra que esa medida 
transfiere los costos ambientales de la producción porcina a áreas dotadas de bosques y pastizales naturales (Bai et al., 2021). Además, obligó a las granjas de traspatio que no podían afrontar los costos de la relocalización o las multas por incumplimiento de las normativas ambientales a abandonar la actividad (Global Times, 2018).

En contraste, se mantuvo el impulso a la modernización del sector porcino. El plan quinquenal para la producción porcina 2016-2020 incluyó como metas el establecimiento de industrias modernas para la cría de cerdos. Además, alentó el desarrollo de las industrias de piensos y veterinaria, la estandarización de la producción y la gestión moderna de las granjas a gran escala (SAIN, 2016).

\section{Cerdos, seguridad alimentaria y peste porcina africana}

La seguridad alimentaria es un asunto de máxima prioridad para China (State Council of the People's Republic of China, 2019). Ahora bien, esa noción es equiparada a la seguridad de granos, lo que tiene como pilar la autosuficiencia (Zha \& Zhang, 2013). China condensó esa aproximación en la estrategia de seguridad alimentaria que estableció el abastecimiento doméstico del $95 \%$ de los granos, mientras que para maíz, arroz y trigo ese nivel debe alcanzar el 100\% (Zhang \& Cheng, 2016).

De todos modos, el logro de esa meta es condicionado por el cambio en la dieta de la población china hacia un creciente consumo de proteínas, lo que forzó al gobierno a reconocer la importancia fundamental de alimentos no básicos, entre ellos, la carne de cerdo, en la seguridad alimentaria (Tortajada \& Zhang, 2021). Esto no resulta extraño dado el ascenso del consumo per cápita anual de carne porcina desde $8 \mathrm{~kg}$ en 1980 a $39 \mathrm{~kg}$ en 2013 (Trápaga Delfín, 2017). Ese nivel de consumo demuestra el éxito del gobierno por satisfacer las expectativas de ingesta de carne de los sectores urbanos, lo que es esencial, puesto que el abastecimiento alimentario a precios estables constituye una fuente de legitimidad política y estabilidad social (Zhang \& Cheng, 2016). 
Por consiguiente, China privilegió la producción doméstica para el abastecimiento de cerdos. Las importaciones porcinas solo representaron un 1,8\% de la demanda total en 2015 (Fok \& Hui, 2017), aunque ello no debe enmascarar que China es el mayor importador mundial de cerdo, lo que refleja su colosal nivel de consumo. No obstante, la autosuficiencia porcina queda relativizada al considerar su dependencia de las importaciones de soja y maíz (Schneider, 2014).

El reporte del primer caso de PPA en la provincia de Liaoning, en agosto de 2018, puso bajo inusitada presión a la industria porcina china y la estrategia de seguridad alimentaria. La PPA es una enfermedad altamente contagiosa ${ }^{3}$ y de elevada mortalidad que afecta a los cerdos y no posee tratamientos ni vacunas. Rápidamente se hizo realidad la advertencia de la FAO (2017) respecto a las devastadoras consecuencias del ingreso de la PPA a China para la salud animal, la seguridad alimentaria y su alta posibilidad de diseminarse. A pesar de las medidas del gobierno para contener la enfermedad, ${ }^{4}$ hacia mediados de 2019, todas las provincias chinas habían registrado brotes de PPA. Asimismo, se expandió a Corea del Sur, Corea del Norte, Mongolia, Camboya, Indonesia, Laos, Myanmar, Filipinas, Timor Leste y Vietnam, así como a países de África y Europa Central (OECD/ FAO, 2020).

Los sucesivos brotes tuvieron un efecto catastrófico en la industria porcina china. La muerte masiva de animales infectados y los sacrificios preventivos (Haley \& Gale, 2020) diezmaron las piaras. Se estima que hacia mediados de 2019 se habían infectado entre 150 y 200 millones de cerdos, lo que supone el $30 \%$ de la población porcina de China (Mason-D'Croz et al., 2020). Otras estimaciones indican que las pérdidas pueden representar hasta el $55 \%$ de las piaras del

\footnotetext{
3 Se transmite por contacto directo con animales infectados e indirectamente por el consumo de desperdicios de alimentos, carne o forrajes infectados o a través de objetos, materiales y transportes que estuvieron en contacto con cerdos contagiados o sus fluidos (Haley \& Gale, 2020).

4 Entre esas medidas se encuentran los sacrificios, el establecimiento de zonas de cuarentena, restricciones en el transporte de carne de cerdo y animales en las áreas con PPA y la prohibición de la alimentación con desperdicios (Hayes et al., 2020).
} 
país e incluso niveles más altos (Weaber \& Habib, 2020). Además, la amenaza para la seguridad alimentaria de China que supuso la drástica reducción de la oferta porcina se vio exacerbada por el aumento de los precios del cerdo de 25 RMB en el periodo previo a la PPA a 60 RMB en octubre de 2019 (Ma et al., 2021).

No obstante, antes que cuestionar el modelo de producción que originó esa situación, la respuesta del gobierno se dirigió a resguardar y fortalecer la industria porcina china de dos formas. Por un lado, mediante el lanzamiento de un plan para estabilizar, recuperar y estimular la producción porcina doméstica a niveles pre-PPA para fines de 2020. Este incluyó el financiamiento para el desarrollo de granjas a gran escala y el estímulo al desarrollo de una vacuna y asesoramiento en el control de la PPA. La OECD y la FAO (2020) alertaron que esas medidas conducirían a una mayor concentración del sector y la desaparición de las granjas pequeñas, que recibieron subsidios para sacrificar sus rebaños.

Por otro lado, la escasez doméstica provocó un incremento de importaciones de cerdo, que entre 2018 y 2019 crecieron un 70\% (OECD/FAO, 2020). Entonces, pese a sus consecuencias negativas, la PPA se convirtió en una oportunidad para países productores y empresas procesadoras interesadas en el mercado chino, así como para empresas chinas que decidieron importar cerdos para beneficiarse de los altos precios domésticos. ${ }^{5}$ Con todo, las importaciones representaron menos del 5\% del total consumido en China (Ma et al, 2021). Un factor que limitó la contribución de las compras externas al abastecimiento doméstico fue la propagación de la COVID-19. La causa fueron las interrupciones y demoras en la distribución asociadas a la reducción de la mano de obra en las plantas procesadoras y los procedimientos de inspección y cuarentena. China también prohibió las importaciones de alimentos con presencia de coronavirus (Ma et al, 2021; Torjadada \& Zhang, 2021).

\footnotetext{
Ejemplo de ello es la firma de un memorando de entendimiento entre WH Group y JBS para la distribución de productos bovinos, porcinos y avícolas frescos al mercado chino (GRAIN, 2020).
} 
El contexto de sostenida expansión de la PPA, su simultaneidad con la COVID-19 y la urgencia por suplir la demanda determinaron que en 2020 la Comisión Nacional de Desarrollo y Reforma y el Ministerio de Agricultura de China publicaran un comunicado que alentaba la producción porcina de las empresas domésticas en el exterior. Los destinos privilegiados para esas operaciones son países que posean vínculos económicos con China, habilitados a exportar cerdos a ese mercado y libres de PPA (CGTN, 2020). Cabe destacar que esta estrategia estaba siendo explorada por la empresa New Hope Liuhe, que construyó una granja porcina de 300000 cerdos en Vietnam (China Knowledge, 2019). Otro ejemplo es el interés de inversores de la provincia de Jiangxi en la construcción de una granja de 100000 cerdos en Belarús para exportar a China (Belarus.by, 2018).

Por consiguiente, lo que se podría calificar como un go out porcino abrío otra vía por la que la industria porcina China puede reconfigurar los mercados alimentarios no solo por las importaciones de soja y maíz, sino también mediante el traslado de su producción de cerdos al exterior. A pesar de sus consecuencias sanitarias, ambientales y sociales, estas inversiones no marcan una transformación de la lógica de producción agroalimentaria hegemónica, sino su expansión hacia otras latitudes.

\section{China y el establecimiento de granjas porcinas en Argentina}

El boom en la demanda de soja de China motorizó una intensificación de sus vínculos con Argentina. En el siglo XXI, China se convirtió en el primer destino para las exportaciones agrícolas argentinas, dominadas por la soja, que representa el $60 \%$ de las ventas al gigante asiático (Sevares, 2018). A ello se añade el ingreso de préstamos e inversiones chinas, concentradas en infraestructura ligada al acceso y transporte de recursos naturales (Laufer, 2020). De esa forma, Argentina encontró en China no solo a un socio comercial clave, sino también una fuente de capital ante la exclusión de los mercados internacionales de crédito (Mora, 2019). Ello favoreció que entre sectores políticos 
y económicos se instalase la creencia en que los lazos con China son la única vía para el desarrollo (Slipak, 2014). Esa visión oculta que el fortalecimiento de la relación con China contribuyó a la exacerbación del extractivismo en Argentina, lo que implica que se fortaleció la primacía de la explotación intensiva de la naturaleza y la exportación de materias primas en la organización de las estructuras económicas, socioterritoriales y de poder (Machado Araoz, 2013).

Por lo tanto, aunque Argentina era un eje de la industria porcina china de modo indirecto a través de las exportaciones de soja, tras el brote de PPA ambos Estados intentaron que esa se transformara en una participación más activa. Con ese fin, en 2019 se acordó la apertura del mercado chino para la carne de cerdo argentina y se habilitaron tres plantas exportadoras (MAGyP, 2019b). Sin embargo, el dinamismo de los vínculos y la relevancia de los alimentos en estos, así como las condiciones sanitarias y ambientales convirtieron al extenso territorio sudamericano en un destino clave para la externalización de la producción china de cerdo. Esta situación convergió con la delicada situación financiera de Argentina tras el endeudamiento con el Fondo Monetario Internacional (FMI) en 2018.

La iniciativa porcina adquirió estado público mediante un comunicado emitido por la Cancillería Argentina en julio de 2020. El texto mencionaba la existencia de negociaciones entre el gobierno argentino y el Ministerio de Agricultura de China para la producción porcina mediante inversiones mixtas, que contaban con el acuerdo de la Asociación China para la Promoción Industrial y la Asociación Argentina de Productores Porcinos. Asimismo, se afirmaba que "la Argentina podría producir 9 millones de toneladas de carne porcina de alta calidad y le daría a China absoluta seguridad de abastecimiento durante muchos años" (Ministerio de Relaciones Exteriores, Comercio Internacional y Culto [MRECIC], 2020a), lo que expresaba claramente que el proyecto suponía un offshoring de la industria porcina de la potencia asiática hacia el país sudamericano. No obstante, posteriormente esa oración fue reemplazada por "La Argentina alcanzará 900 mil toneladas en cuatro años y en un proceso prudente, supervisando las buenas prácticas e incorporando tecnología de punta para reducir 
el impacto medioambiental" (MRECIC, 2020b). Si bien se argumentó que esa modificación se debió a un error, la causa sería la fuerte preocupación social que despertó el anuncio.

Debido a que la Cancillería reiteró en varias oportunidades que el memorándum de entendimiento estaba en elaboración, los documentos oficiales del proyecto no fueron difundidos. Por eso los principales detalles del emprendimiento fueron conocidos mediante entrevistas y declaraciones de prensa de funcionarios, mayormente motivadas por inquietudes sociales respecto al impacto ambiental. De acuerdo a esas fuentes, los tres ejes de organización del proyecto serían el desarrollo tecnológico de la producción porcina; la sanidad y el bienestar animal, y la promoción de acuerdos específicos de asociación entre productores argentinos e inversores chinos (MRECIC, 2020c).

Por otra parte, a fin de alcanzar el nivel de producción señalado, se propuso la instalación de 25 granjas integradas de 12000 madres, que contarían con plantas de elaboración de forrajes, biodigestores, criaderos de ciclo completo, frigoríficos y oficinas de la Aduana y del Servicio Nacional de Sanidad y Calidad Agroalimentaria (SENASA). Esto posibilitaría incorporar entre 30000 y 350000 madres en cuatro años. El destino privilegiado de esos establecimientos serían las provincias productoras de maíz del norte y del sur del país (Cabral, 8 de agosto de 2020). Notoriamente, esta información es coincidente con el documento Complejo tecnológico exportador porcino 20-30 del Ministerio de Agricultura argentino, difundido por la prensa y organizaciones ambientales (Eleisegui, 2020). Ese documento, que presenta el mayor proyecto porcino de Argentina, precisa que las granjas, de un mínimo de 100 hectáreas, se instalarán en zonas que cuenten con capacidad de siembra de 12000 hectáreas de maíz y 5000 de soja; disponibilidad de 1500000 litros de agua diarios; terreno consolidado no inundable en los últimos 50 años, e infraestructura energética y de transporte.

Las características del proyecto indican que apuntaba a replicar en Argentina el modelo de producción porcina industrial presente en China. Un primer elemento a considerar es que Argentina posee una existencia de 5291453 cerdos, y en 2019 produjo 629854 toneladas de esa carne 
(MAGyP, 2019a). Por lo tanto, la expansión de la producción proyectada en las negociaciones con China en un breve lapso solo sería posible mediante un modelo de cría intensiva a gran escala. Evidencia de ello es que alcanzar la cifra inicial de 9 millones de toneladas requeriría pasar a una producción de 100 millones de cerdo en un plazo de 5 a 8 años (Grupo Insud, 2020). Aunque esas metas fueron reducidas, alcanzar una producción adicional de 900000 toneladas igualmente exigiría incorporar casi 10 millones de cabezas (FARN, 2020). Tampoco modifica el hecho que la producción tendrá lugar en megagranjas que integrarán desde la elaboración de forrajes hasta la exportación.

Si bien Argentina había expresado su interés en modernizar el sector porcino para aprovechar la demanda de China (MAGyP, 2019c), el acuerdo porcino revela que, en el logro de ese objetivo, la dimensión ambiental es subordinada a los imperativos de eficiencia y productividad. De hecho, la Cancillería reconoció que las negociaciones se desarrollaban en ausencia de consultas con los Ministerios de Ambiente y Salud (MRECIC, 2020c). La exclusión de estos organismos en la formulación de una iniciativa de esa escala de producción, asociada a una industria cuyos daños ambientales y sobre la salud fueron alertados por diversas organizaciones internacionales (FAO et al., 2010; FAO et al., 2020) refuerza las ideas y prácticas que fundamentan el modelo agroalimentario hegemónico.

De modo similar, tampoco hubo consultas con la sociedad, aunque ello no impidió que esta hiciera oír su voz. La difusión del proyecto provocó un inesperado y contundente rechazo por parte de agrupaciones sociales, ambientales y animalistas, a las que se unieron académicos, artistas, partidos políticos, productores agropecuarios, ciudadanos, etc. A través de comunicados, movilizaciones multitudinarias a lo largo del país y campañas en redes sociales, estos sectores de la sociedad civil desempeñaron un papel fundamental no solo en el conocimiento público de la iniciativa sino que, en su rechazo a que "Argentina se convierta en una factoría de cerdos para China (o para quien sea)" (Organizaciones, agrupaciones y ciudadanos de Argentina, 2020), contribuyeron además a visibilizar y colocar en discusión 


\section{0 / SOL MORA}

otras consideraciones, más allá de las meramente económicas, que subyacen a emprendimientos de este tipo.

Así, en un contexto en que Argentina se encontraba atravesando los graves costos y desafíos impuestos por la pandemia de COVID-19, las dimensiones sanitarias y socioambientales de las megagranjas adquirieron gran trascendencia social. La crueldad e insostenibilidad que supone la ganadería industrial llevaron a que esta fuera considerada "una incubadora de nuevos virus altamente contagiosos y, por ende, en fábricas de nuevas pandemias" (Organizaciones, agrupaciones y ciudadanos de Argentina, 2020). Esa preocupación surgió del riesgo de resistencia bacteriana asociada a la cría industrial de animales en confinamiento y emergencia de zoonosis con potencial pandémico (Karesh et al., 2012; FAO, 2016; Bueno, 2020; Verzeñassi et al., 2020).

Lo anterior se combina con el alto consumo de agua que conlleva la producción porcina a gran escala, estimado en unos 12000 millones de litros, sin incluir la demanda para limpieza y los efluentes (FARN, 2020). A ello se añaden los niveles extraordinarios de contaminación del agua y los suelos a causa de los excrementos de los animales, que se acumulan en piletones, lo que produciría olores nauseabundos que deteriorarían la calidad de vida (Barruti et al, 2020). También se señalaron los efectos de esa degradación del aire, el agua y el suelo sobre la salud humana (Verzeñassi et al, 2020).

Otro aspecto que resultó relevante fue el previsible incremento de la demanda de soja y maíz que exigiría el aumento de la producción porcina. Las estimaciones sugieren que conduciría a la incorporación de casi 290000 hectáreas adicionales de maíz y 250000 hectáreas de soja (FARN, 2020). Ese avance de la frontera agropecuaria aceleraría la deforestación e impulsaría desalojos de campesinos y pueblos originarios (Greenpeace, 2020). Más aún, esos territorios se verían sujetos a las presiones ambientales derivadas del uso de agrotóxicos y fertilizantes para la producción intensiva de soja transgénica. En consecuencia, el emprendimiento supone una profunda reestructuración de los territorios que no solo robustecería el modelo de agronegocios vigente en el país, sino que exacerbaría los efectos ambientales, 
sanitarios y sociales negativos de este modelo como resultado de su combinación con la producción porcina a gran escala.

A causa de los factores mencionados, los movilizados señalaron que la instalación de las granjas conduciría a una duplicación de las emisiones de GEI (FARN, 2020). Notoriamente, los beneficiarios de los múltiples costos sanitarios y ambientales del proyecto serían las grandes empresas del agronegocio argentinas y chinas. Por el contrario, los pequeños y medianos productores porcinos denunciaron que la iniciativa, que era negociada sin considerar sus necesidades y opiniones, amenazaba la agricultura familiar puesto que reforzaba la concentración de la producción y la expulsión de los productores de menor escala debido a la ausencia de créditos y la baja rentabilidad de la actividad (Foro Nacional por un Programa Agrario Soberano y Popular, 2020).

En cambio, este sector formuló alternativas para incrementar la producción porcina con base en la agricultura familiar, que contemplen el bienestar animal y el respecto al ambiente. Por ejemplo, la propuesta de la Federación de Cooperativas Federadas, la Unión de Trabajadores de la Tierra, el Movimiento Nacional Campesino-Indígena y el Frente Nacional Campesino (2020) incluía el apoyo estatal para multiplicar el número de pequeños productores con sistemas al aire libre. Además de requerir baja inversión en capital y generar puestos de trabajo, eso permitiría obtener un producto diferenciado, cuya demanda no se modificaría tras la recuperación de la producción doméstica de China. En sintonía, la Federación Agraria Argentina —FAA_ (2020) destacó que Argentina debía desarrollar su propio plan porcino, que fomentara la producción a pequeña escala en todo el territorio.

El amplio debate en torno a las consecuencias ambientales de la iniciativa tuvo la fuerza suficiente para que la Cancillería postergara la firma del memorándum con el objeto de añadir un artículo referente al respeto de las leyes ambientales. De hecho, los argumentos ambientales pasaron a formar parte del discurso del gobierno argentino frente al proyecto, que incluso declaró que las granjas a instalar serían inteligentes, seguras y sostenibles, diferentes a las megafactorías 


\section{I SOL MORA}

de Estados Unidos (Agencia Telam, 2020). Sin embargo, persisten temores de que estas decisiones solo busquen atenuar las resistencias que dificultan la puesta en marcha del proyecto. $\mathrm{Al}$ respecto, aunque aún no trascendió información sobre la firma del acuerdo, parecería que las provincias encabezarán la ejecución del plan. Muestra de ello es el acuerdo de Chaco con la empresa Feng Tian Food para la instalación de tres complejos integrados para la producción porcina para exportación. Cada complejo contará con cinco granjas de 2400 madres cada una (Diario Chaco, 2020). Por otra parte, mientras que La Rioja concedió un crédito de 200 millones a la empresa porcina de capitales estatales Cerdos de los Llanos para expandir sus plantas (El Intransigente, 2021), Tucumán incluyó el desarrollo de un polo porcino y la mejora de los frigoríficos como parte de su Plan de Obras Públicas (El Tucumano, 2020). Aunque en los últimos casos aún no es explícita su adhesión a la iniciativa porcina con China, esas acciones pueden interpretarse como muestras del interés por participar en esta.

\section{Conclusiones}

La epidemia de PPA que golpeó a la industria porcina china reivindicó la estrecha vinculación entre modelos productivos, ambiente y salud. Al mismo tiempo, fue el preludio de la centralidad que adquirirá esa interrelación durante la pandemia de COVID-19. La rápida difusión de la PPA a lo largo de China, así como posteriormente a otros países, demuestra la fragilidad y crueldad que subyace a un modelo de producción industrial, basado en el confinamiento de animales a gran escala que derivó en la muerte de millones de cerdos. Ahora bien, el estudio del proyecto porcino en Argentina demuestra la apuesta decidida de China por ese modelo de porcicultura industrial mediante su traslado al exterior.

Por consiguiente, la iniciativa en Argentina es inescindible y a la vez fortalece las lógicas constitutivas del sistema agroalimentario global. Por eso debe ser comprendida como una respuesta de urgencia a la necesidad de China de suplir la escasez doméstica de cerdos a fin de mantener su primacía global en la producción y consumo de esa carne. 
Con todo, es importante destacar que es el alto consenso en torno a los supuestos eficientistas, productivistas y economicistas que rigen el modelo agroalimentario hegemónico lo que provocó que la PPA se convirtiera en una oportunidad de lucro no solo para empresas de China, sino también para diferentes niveles del gobierno y actores ligados al agronegocio en Argentina. Ello ocurrió obturando la discusión en torno a la faceta sanitaria y ambiental constitutiva a la actividad porcina industrial y las consecuencias derivadas de la intensificación y extensión de esas prácticas a nuevos territorios.

El intento por robustecer el actual sistema agroalimentario representa una contradicción cuando su carácter destructivo queda expuesto con la crisis ambiental y sanitaria que afecta a todo el globo con repercusiones en múltiples esferas, entre ellas la económica. Justamente, la PPA y la COVID-19 ponen de relieve la inviabilidad de las visiones sectoriales y cortoplacistas que solo valorizan a la naturaleza por su contribución al crecimiento económico. Por el contrario, la emergencia de ambas enfermedades expresa la imposibilidad de escindir el bienestar humano, animal y ecosistémico, y, por ende, que es urgente un sistema agroalimentario sustentado en nuevas ideas y prácticas. De ahí que el amplio rechazo social que despertó el proyecto porcino no solo reclame la relevancia que posee la transformación del sistema agroalimentario, sino que es una muestra del potencial de la sociedad para condicionar iniciativas que fortalezcan ese sistema.

\section{Referencias}

Agencia Telam. (17 de diciembre de 2020). Argentina ratificó la propuesta de instalar granjas inteligentes de producción porcina. Agritotal.com. https://www.agritotal.com/nota/46262-argentina-ratifico-la-propuesta-de-instalar-granjas-inteligentes-de-produccion-porcina/

Bai, Z., Zhao, J., Wei, Z., Jin, X., \& Ma, L. (2019). Socioeconomic drivers of pig production and their effects on achieving sustainable development goals in China. Journal of Integrative Environmental Sciences, 16(1), 141-155. https:// doi.org/10.1080/1943815X.2019.1671463 
Bai, Z., Jin, X, Oenema, O., Lee, M., Zhao, J., \& Ma, L. (2021). Impacts of African swine fever on water quality in China. Environmental Research Letters, 16. https://doi.org/10.1088/1748-9326/abe2ce

Barruti, S., Bonomo, I., Colombo, R., Filardi, M., Folguera, G., Svampa, M., \& Viale, E. (2020). 10 mitos y verdades de las megafactorias de cerdos que buscan instalar en Argentina. https:/ /www.biodiversidadla.org/content/ download/168673/1247721/file/10+mitos+y+verdades + de + las + m egafacorias + de + cerdos $+-+8+$ oct $+2020+-+$ monadanomada + .pdf

Belta. (17 de agosto de 2018). Chinese company ready to invest in large-scale pig farming in Minsk Oblast. Belta. https:/ / eng.belta.by/economics/ view/chinese-company-ready-to-invest-in-large-scale-pig-farming-inminsk-oblast-114095-2018

Bueno, M. de P. (2020). Cuando la receta del éxito redunda en degradación y pandemia: de cerdos y capitalismos. Temas y Debates, 24(Número Especial), 477-485. https://doi.org/10.35305/tyd.v0i0.524

Cabral, F. (8 de agosto de 2020). Argentina presentará una propuesta para reformular el arancel externo común del Mercosur. TELAM Digital. https://www.telam.com.ar/notas/202008/499962-arancel-externo-mercosur-jorge-neme-porcinos-china-entrevista.html

CGTN. (18 de marzo de 2020). China releases new measures to support hog farming, encourages firms to raise pigs abroad. https:// news.cgtn.com/ news/2020-03-18/China-says-it-supports-companies-to-build-pigfarms-abroad-OXt1LPhvck/index.html

China Knowledge. (28 de agosto de 2019). China's New Hope Liube completed its first overseas pig farm in Vietnam. https://m.chinaknowledge.com/ News/DetailNews/87842/China\%E2\%80\%99s-New-Hope-Liuhecompleted-its-first-overseas-pig-farm-in-Vietnam

Clapp, J., \& Moseley, W. (2020). This food crisis is different: CovID-19 and the fragility of the neoliberal food security order. The Journal of Peasant Studies 47(7), 1393-1417, https:/ / doi.org/10.1080/03066150 .2020 .1823838

Cox, R. (1992). Multilateralism and world order. Review of International Studies, 18(2), 161-180. https://www.jstor.org/stable/20097291

Cox, R. (1993). Gramsci, hegemony and international relations: An essay in method. En S. Gill (Ed.), Gramsci Historical Materialism and International Relations (pp. 49-66). Cambridge University Press. 
Cox, R. (2013). Fuerzas sociales, estados y órdenes mundiales: Más allá de la teoría de las relaciones internacionales. Relaciones Internacionales (24), 129-162. https:// revistas.uam.es/relacionesinternacionales/article/ view/5195

Diario Chaco. (29 de octubre de 2020). Capitanich firmó con una empresa china la puesta en marcha de tres complejos productivos porcinos con destino a exportación. http://www.diariochaco.com/noticia/capitanich-firmo-con-unaempresa-china-la-puesta-en-marcha-de-tres-complejos-productivos

El Intransigente. (20 de enero de 2021). Anuncian inversiones millonarias para la producción de cerdos: ¿Avanza el acuerdo porcino con China? https:/ / elintransigente.com/2021/01/anuncian-inversiones-millonarias-para-laproduccion-de-cerdos-avanza-el-acuerdo-porcino-con-china/

El Tucumano. (14 de agosto de 2020). Tucumán presentó a Nación su Plan de Obras de infraestructura. https://www.eltucumano.com/noticia/ actualidad/265937/tucuman-presento-a-nacion-su-plan-de-obrasde-infraestructura

Eleisegui, P. (26 de agosto de 2020). Argentina será la granja porcina de China: este es el proyecto del Gobierno para asegurarse millones. IProfesional. https://www.iprofesional.com/negocios/322243-argentinasera-la-granja-porcina-de-china-el-proyecto-oficial

Emel, J., \& Neo, H. (2011). Killing for profit: global livestock industries and their socio-ecological implications. En R. Peet, P. Robbins \& M. Watts (Eds.), Global political ecology (pp. 67-83). Routledge.

ETC Group. (2019). Platetochnics. Mapping corporate power in big food. https:/ / www.etcgroup.org/files/files/etc_platetechtonics_a4_nov2019_web. pdf

Federación Agraria Argentina [FAA]. (7 de septiembre de 2020). Se necesita un plan porcino desde la producción y para el progreso de todos. https:// coprofam.org/2020/09/07/faa-se-necesita-un-plan-porcino-desde-laproduccion-y-para-el-progreso-de-todos /

Food and Agriculture Organization [FAO]. (2014). Tackling climate change through livestock. http://www.fao.org/ag/againfo/resources/en/publications/tackling_climate_change/index.htm

Food and Agriculture Organization [FAO]. 2016. Drivers, dynamics and epidemiology of antimicrobial resistance in animal production. https://www.fao. org/3/i6209e/i6209e.pdf 
Food and Agriculture Organization [FAO]. (2017). African swine fever threatens People's Republic of China. FAO Animal Health Risk Analysis-Assessment, (5).

FAO, IFAD, UNICEF, WFP, \& WHO (2020). The State of Food Security and Nutrition in the World 2020. Transforming food systems for affordable healthy diets. https://doi.org/10.4060/ca9692en

FAO, OIE, \& WB (2010). Good practices for biosecurity in the pig sector - Issues and options in developing and transition countries. FAO Animal Production and Health Paper (169). http://www.fao.org/3/i1435e/ i1435e.pdf

FAO, OIE, \& WHO (2020). FAO/OIE/WHO tripartite statement on the pandemic risk of swine influenza. https://www.3tres3.com/3tres3_common/ art/3tres3/45464/fitxers/FAO $\% 20 O I E \% 20 W H O \% 20$ Tripartite $\% 20$ Statement.pdf

FAO, OIE, WHO, UN System Influeza Coordination, UNICEF, \& WB. (2008). Contributing to one world, one health. A strategic framework for reducing risks of infectious diseases at the animal-buman-ecosystems interface. https:// www.fao.org/3/aj137e/aj137e00.htm

Fundación Ambiente y Recursos Naturales [FARN]. (2020). ¿Cerdos para China made in Argentina? Acerca del posible acuerdo de producción y exportación de carne porcina a la República Popular de China. https:// farn.org. ar/cerdos-para-china-made-in-argentina/

FECOFE, MNCI-ST, UTT, \& FNC. (8 de octubre de 2020). Propuestas para una producción porcina sustentable: no a las megagranjas para China. Biodiversidad LA. https:/ /www.biodiversidadla.org/Campanasy-Acciones/Propuestas-para-una-produccion-porcina-sustentable-noa-las-megagranjas-para-China

Fok, A., \& Hui, A. (2017). China pork sector. Asian Insights SparX. DBS Group Research. https://www.dbs.com.sg/sme/aics/pdfController. page?pdfpath=/content/article/pdf/AIO/052017/170510_insights_ opportunities_arising_in_china_pork_sector.pdf

Foro Nacional por un Programa Agrario Soberano y Popular. (2020). Sobre el acuerdo con China para la cría industrial de cerdos para la exportación. http:/ / foroagrario.org/sobre-el-acuerdo-con-china-para-la-cria-industrialde-cerdos-para-la-exportacion/ 
Giudice, F, Caferra, R., \& Morone, P. (2020). CovID-19, the food system and the circular economy: Challenges and opportunities. Sustainability 12 (19), 1-15 https:/ / doi.org/10.3390/su12197939

Global Times. (10 de enero de 2018). Afterwater, China's pigs join south-to-north transferprojects to address environmental concerns. https: / / www.globaltimes. $\mathrm{cn} /$ content/1084207.shtml

Gooch, E., \& Gale, F. (2018). China's foreign agriculture investments. Economic Information Bulletin, (192). https://www.ers.usda.gov/webdocs/ publications/88572/eib-192.pdf

GRAIN. (2020). Peste porcina africana: Un futuro cultivado en granjas industriales, una pandemia a la vez: https://grain.org/es/article/6429-peste-porcinaafricana-un-futuro-cultivado-en-granjas-industriales-una-pandemiaa-la-vez

Gras, C., \& Hernández, V. (2013). Los pilares del modelo agribusiness y sus estilos empresariales. En Autores, El agro como negocio:producción, sociedad $y$ territorios en la globalización (pp. 17-46). Biblos.

Greenpeace. (2020). La instalación de granjas industriales de cerdos en Santiago del Estero generará más desmontes y desalojos de campesinos. https://www. greenpeace.org/argentina/story/issues/bosques/la-instalacion-degranjas-industriales-de-cerdos-en-santiago-del-estero-generara-masdesmontes-y-desalojos-de-campesinos /

Grupo Insud. (10 de enero de 2020). Biogénesis Bagó, impulsora de un importante acuerdo con China. https://www.grupoinsud.com/ biogenesis-bago-impulsora-de-un-importante-acuerdo-con-china/

Haley, M., \& Gale, F. (3 de febrero de 2020). African swine fever shrinks pork production in China, swells demand for imported pork. United States Department of Agriculture. Economic Research Service. https://www.ers.usda. gov/amber-waves/2020/february/african-swine-fever-shrinks-porkproduction-in-china-swells-demand-for-imported-pork/

Hayes, D.; Ji, Y.; Li, M.; Shao, Y., \& Zhang, W. (2020). World's largest pork producer in crisis: China's African swine fever outbreak. Agricultural Policy Review. https://www.card.iastate.edu/ag_policy_review/ article $/ ? \mathrm{a}=85$

Ioris, A. (2018). The politics of agribusiness and the business of sustainability. Sustainability, 10(1648), 1-21. https://doi.org/10.3390/su10051648

International Panel on Climate Change. (2019). Climate change and land: An IPCC special report on climate change, desertification, land degradation, sustainable 
land management, food security, and greenhouse gas fluxes in terrestrial ecosystems. https://www.ipcc.ch/srccl/

James, D., Bowness, E., Robin, T., McIntyre, A., Dring, C., Desmarais, A., \& Wittman, H. (2021). Dismantling and rebuilding the food system after COVID-19: Ten principles for redistribution and regeneration. Journal of Agriculture, Food Systems, and Community Development, 10(2), 29-51. https://doi.org/10.5304/jafscd.2021.102.019

Jin, Z. (15 de julio de 2010). Animal waste a threat to clean water supply. China Daily. http://www.chinadaily.com.cn/china/2010-07/15/content_10108361.htm

Karavas, G., \& Brincat, S. (2016). Behemoth pulls the peasant's plough: Convergence and resistance to business civilization in China. Globalizations, 13(5), 622-637. https://doi.org/10.1080/14747731.2016.1204079

Karesh, W., Dobson, A., O Lloyd-Smith, J., Lubroth, J. Dixon, M., Bennett, M., Aldrich, S., Harrington, T., Formenty,P., Loh, E. , Machalaba, C., Thomas, M., \& Heymann, D. (2012). Ecology of zoonoses: Natural and unnatural histories. The Lancet, 380(9857), 1936-1945. Doi: https:// doi.org/10.1016/S0140-6736(12)61678-X

Lander, B., Schneider, M., \& Brunson, K. (2020). A history of pigs in China: From curious omnivores to industrial pork. 79(4), 865-889. https:// doi.org/10.1017/S0021911820000054

Laufer, R. (2020). El proyecto chino "La Franja y La Ruta” y América Latina: ¿Otro norte para el sur? Revista Interdisciplinaria de Estudios Sociales (20), 9-52 https://ceiso.com.ar/ries/index.php/ojs/article/view/ laufer-ries 20

Lü, M. (25 de marzo de 2013). Shanghai's dead pig story stretches back upstream. China Dialogue. https://chinadialogue.net/en/ pollution/5820-shanghai-s-dead-pig-story-stretches-back-upstream/

Ma, M., Wang, H., Hua, Y., Qin, F., Yang, J. (2021). African swine fever in China: Impacts, responses, and policy implications. Food Policy, 102. https://doi.org/10.1016/j.foodpol.2021.102065

Machado Aráoz, H. (2013). Crisis ecológica, conflictos socio-ambientales y orden neocolonial: las paradojas de nuestra América en las fronteras del extractivismo. Revista Brasileira de Estudos Latino-americanos, 3(1), 118155. https://horizontescomunitarios.files.wordpress.com/2016/10/ machado-araoz-crisis-ecolc3b3gica-conflictos-socioambientales-y-orden-neocolonial.pdf 
Argentina, Ministerio de Agricultura, Ganadería y Pesca [MAGyP]. (2019a). Anuario 2019. Porcinos. https://www.magyp.gob.ar/sitio/areas/porcinos/estadistica/_archivos//000005-Anuario/190000-Anuario\%20 2019.pdf

Argentina, Ministerio de Agricultura, Ganadería y Pesca [MAGyP]. (2019b). La carne de cerdo argentina ingresará al mercado chino. https: / / www.argentina.gob.ar/noticias/la-carne-de-cerdo-argentina-ingresara-al-mercado-chino

Argentina, Ministerio de Agricultura, Ganadería y Pesca [MAGyP]. (2019c). Etchevehere inauguró el primer seminario en conjunto con China sobre carne de cerdo. https:/ / www.argentina.gob.ar/noticias/ etchevehere-inauguroel-primer-seminario-en-conjunto-con-china-sobre-carne-de-cerdo

Mason-D’Croz, D., Bogard, J. R., Herrero, M., Robinson, S., Sulser, T., Wiebe, K., Willenbockel, D., \& Godfray, C (2020). Modelling the global economic consequences of a major African swine fever outbreak in China. Nature Food, 1, 221-228. https://doi.org/10.1038/ s43016-020-0057-2

McMichael, P. (2009). A food regime genealogy. The Journal of Peasant Studies, 36(1), 139-169. https://doi.org/10.1080/03066150902820354

McMichael, P. (2020). Does China's 'going out' strategy prefigure a new food regime? The Journal of Peasant Studies, 47(1), 116-154. https:// doi.org/10.1080/03066150.2019.1693368

Mora, S. (2019). El Going Out agrícola de China. Un análisis de su desarrollo en Argentina. Si Somos Americanos. Revista de Estudios Transfronterizos, 19(2), 89-113. https://doi.org/10.4067/S0719-09482019000200089

Mora, S (2021): Land grabbing, power configurations and trajectories of China's investments in Argentina. Globalizations. https://doi.org/10. 1080/14747731.2021.1920197

Argentina, Ministerio de Relaciones Exteriores, Comercio Internacional y Culto [MRECIC]. (2020a). Diálogo con el ministro de Comercio de la República Popular China Zhong Shan. https://www.cancilleria.gob.ar/es/actuali$\mathrm{dad} /$ noticias/dialogo-con-el-ministro-de-comercio-de-la-republicapopular-china-zhong-shan

Argentina, Ministerio de Relaciones Exteriores, Comercio Internacional y Culto [MRECIC]. (2020b). Entrevista entre el canciller Felipe Solá y el ministro de Comercio de la República Popular China Zhong Shan. https:/ /www. 
cancilleria.gob.ar/es/actualidad/noticias/entrevista-entre-el-cancillerfelipe-sola-y-el-ministro-de-comercio-de-la

Argentina, Ministerio de Relaciones Exteriores, Comercio Internacional y Culto [MRECIC]. (2020c) Jorge Neme: "Podemos producir carne porcina con el mejor estándar sanitario". https://www.cancilleria.gob.ar/es/actualidad/ noticias/jorge-neme-podemos-producir-carne-porcina-con-el-mejorestandar-sanitario

OECD/FAO. (2020). OECD-FAO agricultural outlook 2020-2029. https://doi. org/10.1787/1112c23b-en

World Organization for Animal Health [OIE]. (s.f.). One health. https://www. oie.int/en/what-we-do/global-initiatives/one-health/

Organizaciones, agrupaciones y ciudadanos de Argentina (2020). No queremos transformarnos en una factoría de cerdos para China, ni en una fábrica de nuevas pandemias. https://www.biodiversidadla.org/Campanas-y-Acciones/ No-queremos-transformarnos-en-una-factoria-de-cerdos-para-Chinani-en-una-fabrica-de-nuevas-pandemias

Otero, G. (2013). El régimen agroalimentario neoliberal y su crisis: Estado, agroempresas, multinacionales y biotecnología. Antípoda (17), 49-78. http://dx.doi.org/10.7440/antipoda17.2013.04

Pedreño Cánovas, A., Giménez Casalduero, M., \& Ramírez Melgarejo, A. (2021). Cerdos, acumulación y producción de naturaleza barata. Relaciones Internacionales (47), 143-162.

Petetin, L. (2020). The COVID-19 crisis: An opportunity to integrate food democracy into post-pandemic food systems. European Journal of Risk Regulation 11 (Special Issue 2), 326-336. https://doi.org/10.1017/ err.2020.40

Powers, M. (2021). Food and the global political economy. Ethics \& International Affairs, 35(1), 99-117. https://doi.org/10.1017/ S0892679421000058

Qiao, F., Huang, J., Wang, D, Liu, H., \& Lohmar, B. (2016). China’s hog production: From backyard to large-scale. China Economic Review, (38),199-208. http://dx.doi.org/10.1016/j.chieco.2016.02.003

Saguier, M., \& Ghiotto, L. (2018). Las empresas transnacionales: un punto de encuentro para la economía política internacional de América Latina. Desafios, 30(2),159-190. https://doi.org/10.12804/revistas. urosario.edu.co/desafios/a.6222 
Sustainable Agriculture Innovation Network - UK and China [SAIN]. (2016). National Pig Production Development Plan (2016-2020). http:/ / knowledgeshare.sainonline.org/wp-content/uploads/2017/04/National-PigProduction-Development-Plan-2016-2020.pdf

Sanderson Bellamy A., Furness E., Nicol P., Pitt H., \& Taherzadeh A. (2021). Shaping more resilient and just food systems: Lessons from the COVID-19 Pandemic. Ambio 50(4):782-793. https://doi.org/10.1007/ s13280-021-01532-y

Schneider, M. (2014) Developing the meat grab. The Journal of Peasant Studies 41 (4), 613-633. https://doi.org/10.1080/03066150.2014.918959

Schneider, M. (2017). Dragon head enterprises and the state of agribusiness in China. Journal of Agrarian Change, 17(1), 3-21. https://doi. org/10.1111/joac. 12151

Schoenmakers, K. (2020). How China is getting its farmers to kick their antibiotics habit. Nature (586), 960-962. https://doi.org/10.1038/ d41586-020-02889-y

Sevares, J. (2018). Relaciones ALC-China. Oportunidades y desafíos. Voces en el Fénix (67), 87-93. de https://www.vocesenelfenix.com/content/ relaciones-alc-china-oportunidades-y-desafíos

Sharma, S., \& Schlesinger, S. (2017). The rise of bog meat. Brazil's extractive industry. Institute for Agriculture and Trade Policy. https://www.iatp. org/sites/default/files/2017-11/2017_11_30_RiseBigMeat_f.pdf

Silva, J. H. C. S., \& Barbosa, A. da S. (2020). A inserção da agroecologia em um novo sistema alimentar pós COVID-19. Revista Brasileira de Educação Ambiental 15(4), 148-159. https://doi.org/10.34024/revbea.2020. v15.10618

Slipak, A. 2014. América Latina y China : ¿cooperación Sur-Sur o « Consenso de Beijing »? Nueva Sociedad, (250), 102-113.

State Council of the People's Republic of China (2019). Food security in China. http://english.scio.gov.cn/2019-10/14/content_75300394.htm

Tortajada, C., \& Zhang, H. (2021). When food meets BRI: China's emerging Food Silk Road. Global Food Security (29). https://doi.org/10.1016/j. gfs.2021.100518

Trápaga Delfín, Y. (2017). China y América Latina: complementarios en un modelo depredador de producción porcícola. En Autor, América Latina y el Caribe y China: Recursos naturales y medio ambiente 2017 (pp. 97-112). Unión de Universidades de América Latina y el Caribe. 
Verzeñassi, D., Marino, D., Vallini, A., Alonso, L., Burguener, G., Enriquez, L., Fernández, F, Ferrazini, L., Keppl, G., Lasagna, M., \& Möller, V. (2020). La salud hecha un chiquero: informa del impacto de la cría industrial de cerdos en la salud. Fundación Rosa Luxemburgo.

Weaver, T., \& Habib, N. (2020). Evaluating losses associated with African swine fever in the People's Republic of China and neighboring countries. Asian Development Bank (ADB) East Asia Working Paper Series, (27).

Weis, T. (2013). The meat of the global food crisis. The Journal of Peasant Studies 40(1), 65-85. http: / dx.doi.org/10.1080/03066150.2012.752357

Xinzhen, L. (22 de junio de 2020). The second national census on pollution sources. Beijing Review. https:/ / www.bjreview.com/Opinion/202006/ t20200622_800210925.html

Yeboah O., Thomas T., Foster T., \& Fosu E. (2009). Pollution, environmental justice, and the North Carolina pork industry. En E. Nzewi et al. (Eds), Proceedings of the 2007 National Conference on Environmental Science and Technology. Springer. https://doi.org/10.1007/978-0-387-88483-7

Zha, D., \& Zhang, H. (2013). Food in China's international relations. The Pacific Review, 5(26), 455-479. https://doi.org/10.1080/09512748.20 13.842308

Zhang, H., \& Cheng, G. (2016). China's food security strategy reform: An emerging global agricultural policy. En F. Wu y H. Zhang (Eds.), China's global quest for resources. Energy, food and water (pp. 23-41). Routledge.

Zhang, S., Wu, X., Han, D., Hou, Y., Tan, J., Kim, S., Li, D., \& Wang, J. (2021). Pork production systems in China: A review of their development, challenges and prospects in green production. Frontiers of Agricultural Science and Engineering, 8(1), 15-24. https://doi.org/10.15302/JFASE-2020377

Zhou, G., Zhang, W., \& Xu, X. (2012). China's meat industry revolution: Challenges and opportunities for the future. Meat Science, (92), 188196. https://doi.org/10.1016/j.meatsci.2012.04.016

Zhu, Y., Johnson, T., Su, J., Qiao, M., Guo, G., Stedtfeld, R., Hashsham, S., \& Tiedje, J. (2013). Antibiotic resistance genes on Chinese pig farms. Proceedings of the National Academy of Sciences, 110(9), 3435-3440. https:/ / doi.org/10.1073/pnas.1222743110 